\title{
Safety Report Nullification Reason Code
}

National Cancer Institute

\section{Source}

National Cancer Institute. Safety Report Nullification Reason Code. NCI Thesaurus. Code C94072.

A coded value specifying the reason why the adverse event report is nullified. 